\title{
Use of the ultracentrifuge vertical rotor in the detection of rubella-specific IgM on a sucrose density gradient
}

\author{
P. LUTON AND G. L. RIDGWAY
}

From the Department of Clinical Microbiology, University College Hospital, London WC1E6AU, UK

SUMMARY A comparison was made of the performance of swing-out and vertical ultracentrifuge rotors in the detection of rubella-specific IgM on a sucrose density gradient. Tests were performed on 30 sera, of which 11 were found to contain rubella-specific IgM by both methods. The centrifugation time for the swing-out rotor was 16 hours at $35000 \mathrm{rpm}$. This was reduced to 2 hours using the vertical rotor at $50000 \mathrm{rpm}$. Routine use of the vertical rotor would allow a reduction in the time taken to perform the test, increase the number of sera tested each time, and reduce wear on the ultracentrifuge.

The separation of immunoglobulins on a density gradient is a technique widely used in virology laboratories for the detection of rubella-specific IgM (Vesikari and Vaheri, 1968; Caul et al., 1974; Caul et al., 1978). However, the technique as originally described was time consuming. The period of centrifugation required to separate the immunoglobulins was 14-18 hours. Consequently the test had to be performed over a two-day period.

Recently, ultracentrifuge vertical rotors have become available which reorientate the gradient tube contents during centrifugation. The tubes are held vertically in the rotor, and thus during centrifugation the gradient reorientates through $90^{\circ}$ in parallel with the long axis of the centrifuge tube. This effectively reduces the particle path length from the length of the tube to the diameter of the

Received for publication 12 March 1979 tube, and therefore reduces the required centrifugation time. As the rotor slows down, the gradient reorientates through $90^{\circ}$ again back to the horizontal plane (Fig. 1).

This work was carried out to compare the performance of the vertical rotor to that of the swingout rotor and to determine optimum conditions for the use of the vertical rotor in order to reduce the time taken to detect rubella-specific IgM.

\section{Material and methods}

SER A

The 30 sera tested were from patients suspected of having had a recent rubella infection, and all had rubella haemagglutination inhibition (HAI) titres of at least 128. Included with each batch of sera was a positive control serum confirmed as containing rubella-specific IgM by the original density gradient

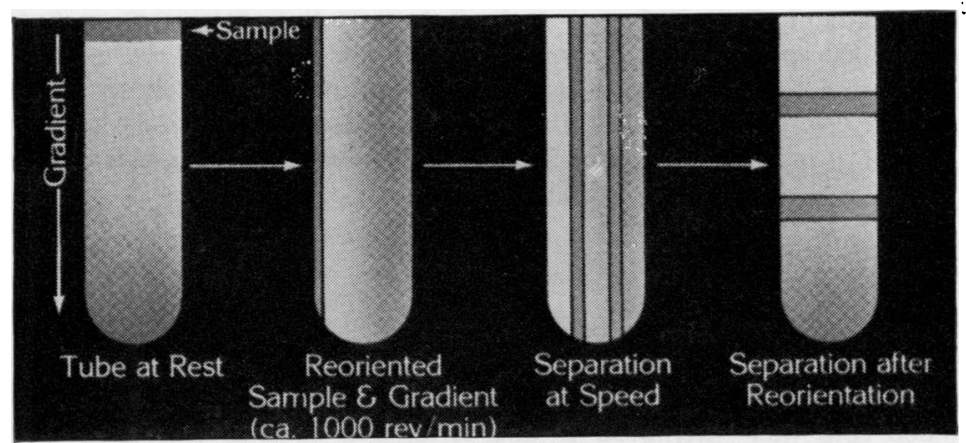

Fig. 1 Density gradient separation in a vertical rotor. 
technique. All the sera were tested using both swing-out and vertical rotors.

PRE-TREATMENT OF SERA

Before centrifugation, $\mathbf{0 . 2 5} \mathrm{ml}$ of serum was added to $0.25 \mathrm{ml}$ of a heparin/manganous chloride mixture prepared by mixing equal volumes of 1000 units $/ \mathrm{ml}$ heparin and $\mathbf{0 . 2} \mathrm{M}$ manganous chloride solution (Pattison and Mace, 1975). The mixture was incubated at $+4^{\circ} \mathrm{C}$ for 20 minutes and then centrifuged at $+4^{\circ} \mathrm{C}$ in an MSE Mistral $6 \mathrm{~L}$ centrifuge at $2000 \mathrm{rpm}$ for 20 minutes. After centrifugation the supernatant was removed and to it was added 1 drop of a $50 \%$ suspension of day-old chick red blood cells (RBCs). This mixture was incubated at $+4^{\circ} \mathrm{C}$ for 1 hour before being centrifuged at $1500 \mathrm{rpm}$ for 5 minutes to remove the RBCs. The supernatant serum was then used for the test.

\section{PREPARATION OF GRADIENTS}

The sucrose solutions used to form the gradients were of the following concentrations: $12 \cdot 5,18 \cdot 75,25$, 31 , and $37.5 \%(\mathrm{w} / \mathrm{v})$ made up in phosphate-buffered saline and stored at $+4{ }^{\circ} \mathrm{C}$. Discontinuous gradients were formed by layering $0.8 \mathrm{ml}$ amounts of each concentration into $5 \mathrm{ml}$ polyallomer ultracentrifuge tubes (Du Pont Instruments), and the $0.5 \mathrm{ml}$ of pretreated serum was layered onto the top of the gradient (Caul et al., 1974). The gradient tubes were then centrifuged as described below.

\section{CENTRIFUGATION USING THE VERTICAL} ROTOR

To determine the approximate centrifugation time required to separate the immunoglobulins, the $\mathbf{K}$ or clearing factors were calculated for both rotors using the formula:

$$
K=253300 \ln \left(\frac{R \text { max }}{R \min }\right) \div\left(\frac{N}{1000}\right)^{2}
$$

where $\mathbf{N}=$ rpm

$\mathbf{R} \max =$ radial distance to the bottom of the tube $\mathbf{R}$ min = radial distance to the top of the tube.

Using the manufacturer's data(Du Pont Instruments), the $\mathrm{K}$ factor for the swing-out rotor at $35000 \mathrm{rpm}$ was calculated to be 113 . For the vertical rotor, the factor was 17 .

$$
\text { Using the formula } T_{v}=T_{s}\left(\frac{K_{v}}{K_{s}}\right)
$$

where $T_{\mathbf{v}}=$ centrifugation time for vertical rotor

$T_{s}=$ centrifugation time for swing-out rotor

$\mathrm{K}_{\mathbf{v}}=\mathrm{K}$ factor for vertical rotor

$\mathbf{K}_{\mathrm{s}}=\mathbf{K}$ factor for swing-out rotor

the time calculated to produce immunoglobulin separation in the vertical rotor at $50000 \mathrm{rpm}$ was
2.4 hours. The gradient tubes were placed into a Sorvall TV-865 $8 \times 5 \mathrm{ml}$ vertical rotor and were centrifuged at $50000 \mathrm{rpm}$ at $+4^{\circ} \mathrm{C}$ in the same OTD-50 ultracentrifuge. Centrifugation times were varied from $1 \frac{1}{2}$ to $2 \frac{1}{2}$ hours in 15 -minute intervals, and the time chosen for routine use was that which gave results similar to the results obtained using the swing-out rotor.

When using either rotor the rate of acceleration up to $1000 \mathrm{rpm}$ was controlled by the automatic rate controller fitted to the ultracentrifuge. During deceleration the braking system was switched off to allow the rotor to coast slowly to a stop.

FRACTION COLLECTION AND TREATMENT Fractions were collected using an MSE tube piercer. Twelve fractions of $0.4 \mathrm{ml}$ were collected from each tube and tested as described below. Fractions 1-6 were also treated with 2-mercaptoethanol (2-ME) by the method of Caul et al. (1974) in order to distinguish IgM from non-specific inhibitors or aggregated IgM (Caul et al., 1978).

\section{RUBELLA HAI TESTS}

The 12 fractions from each gradient were serially double diluted to 1 in 128 . To each dilution was added an equal volume of rubella antigen containing 4 HA units. The mixture was incubated at room temperature for 1 hour before the addition of a volume of $0.4 \%$ day-old chick RBCs. After addition of the cells the tests were incubated at $+4{ }^{\circ} \mathrm{C}$ for 1 hour before being read. The 2-ME treated fractions were similarly tested.

\section{Results}

Of the 30 sera tested, 11 were found to contain rubella-specific IgM by the standard method using a swing-out rotor. The same 11 sera were also found to contain rubella-specific IgM when centrifuged in a vertical rotor. A comparison between the results obtained using the swing-out and vertical rotors is shown in Figure 2. The serum tested was the positive control serum, and the centrifugation time using the vertical rotor was 2 hours. Treatment of the positive control fractions 1-6 from both rotors with 2-ME caused reductions in antibody titre in fractions $2-5$, the greatest reduction being in fractions 3 and 4, as shown in Figure 3.

\section{Discussion}

It can be seen from Fig. 2 that immunoglobulin separation in the vertical rotor with a centrifugation time of 2 hours is virtually identical with that achieved in the swing-out rotor in a time of 16 


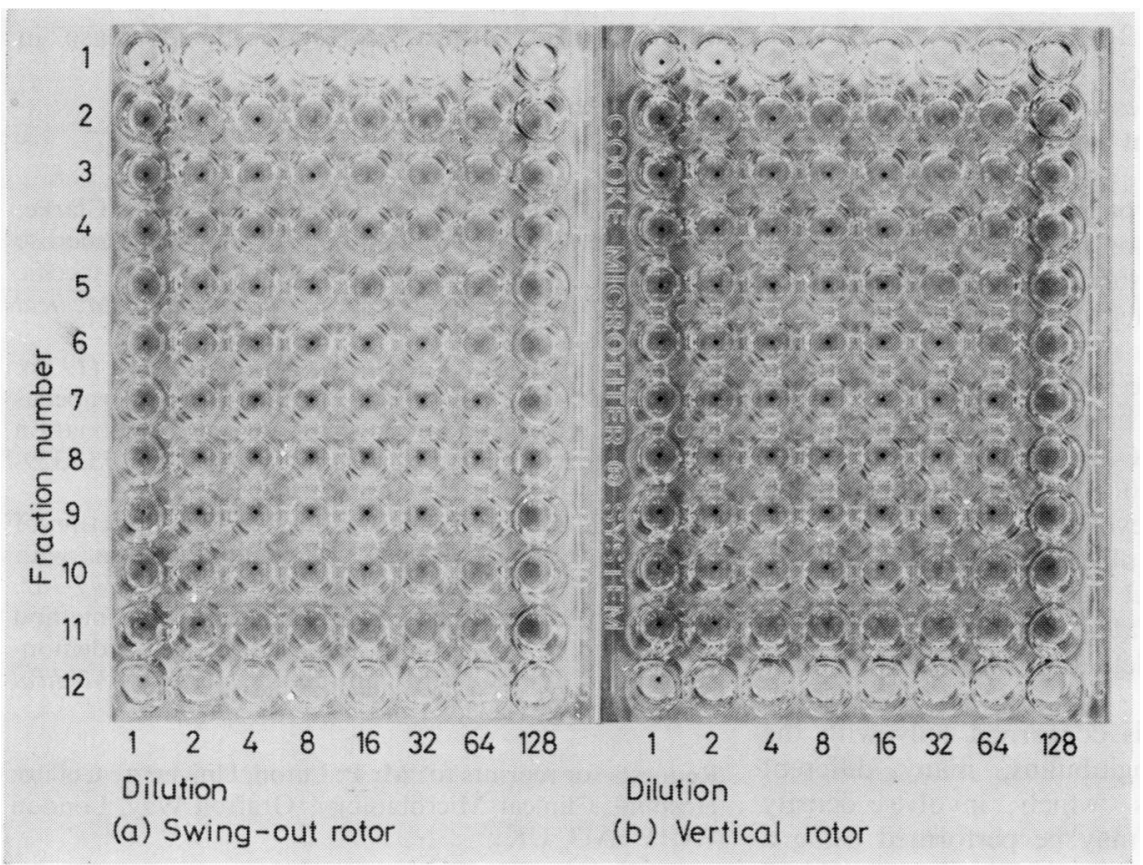

Fig. 2 ( $a$ and $b$ )

Rubella HAI tests of the fractions collected after density gradient centrifugation.

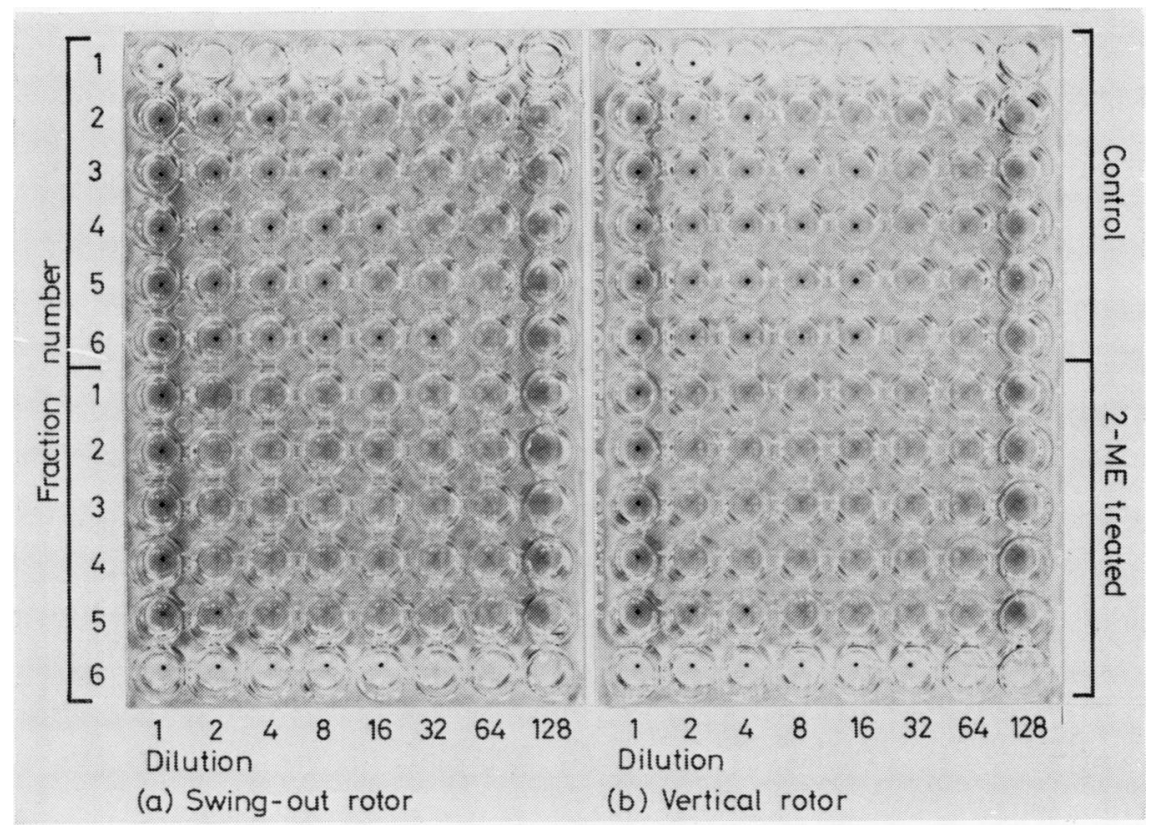

Fig. 3 (a and $b$ )

Rubella HAI tests of fractions $1-6$ before and after treatment with 2-ME.

hours. Figure 3 shows that IgM is at a maximum in fractions 3 and 4 by both methods. It is therefore suggested that a 2-hour centrifugation period is adopted when using the vertical rotor. Once the system has been calibrated with a positive control serum, only the fractions likely to contain IgM need be collected. They can then be tested for the presence of rubella antibodies by HAI both before 
and after treatment with 2-ME (Caul et al., 1974).

Using the conditions described above, it is possible to complete the whole test in one day. This is not practical with a swing-out rotor since even at 50000 rpm the calculated centrifugation time is $\mathbf{7 . 8}$ hours. Also, most swing-out rotors hold only six tubes whereas the vertical rotor (Sorvall TV-865) has a capacity of eight tubes, thereby enabling more sera to be tested in a far shorter time than was previously possible.

Wear on the centrifuge is greatly reduced because during a period of 2 hours at $50000 \mathrm{rpm}$ the centrifuge drive rotates through only 6 million revolutions, while for 16 hours at $35000 \mathrm{rpm}$ the figure is 33-34 million revolutions.

The Sorvall OTD-50 ultracentrifuge will operate at up to $50000 \mathrm{rpm}$, but the TV-865 vertical rotor is capable of being used at up to $65000 \mathrm{rpm}$. At this maximum speed the calculated centrifugation time would be 1 hour.

Although this work is concerned only with the separation of immunoglobulins, many different preparation techniques which involve density gradient centrifugation may be performed using a vertical rotor with a corresponding decrease in centrifugation time.

\section{References}

Caul, E. O., Hobbs, S. J., Roberts, P. C., and Clarke, S. K. R. (1978). Evaluation of a simplified sucrose gradient method for the detection of rubella-specific IgM in routine diagnostic practice. Journal of Medical Virology, 2, 153-163.

Caul, E. O., Smyth, G. W., and Clarke, S. K. R. (1974). A simplified method for the detection of rubellaspecific IgM employing sucrose density fractionation and 2-mercaptoethanol. Journal of Hygiene, 73, 329340.

Pattison, J. R., and Mace, J. E. (1975). The detection of specific IgM antibodies following infection with rubella virus. Journal of Clinical Pathology, 28, 377-382.

Vesikari, T., and Vaheri, A. (1968). Rubella: a method for rapid diagnosis of a recent infection by demonstration of the IgM antibodies. British Medical Journal, 1, 221-223.

Requests for reprints to: Mr P. Luton, University College Hospital, Clinical Microbiology, Grafton Way, London WC1E 6AU, UK. 